\title{
Empreendedorismo e inovação: um retrato do contexto brasileiro dos anos de 2005 a 2011
}

Os temas empreendedorismo e inovação tem sido muito discutido na atualidade, tanto em meios acadêmicos, empresariais quanto políticos. E, embora constituam, aparentemente, conceitos diferentes, sugere-se a existência de uma relação entre eles. Assim, na tentativa de verificar a existência dessa associação, o presente estudo realizou uma pesquisa qualitativa e exploratória, que se utilizou de dados quantitativos. Os resultados da pesquisa mostram que os termos empreendedorismo e inovação, embora tenham alguma proximidade, expressam conceitos diferentes. 0 empreendedorismo consiste no processo por meio do qual indivíduos transformam ideias em oportunidades, a fim de gerar riqueza. A inovação, por sua vez, é o processo por meio do qual novos produtos e serviços ou processos são implementados; também visa à continuidade do negócio e a geração de receita. Assim, a novidade necessária à inovação consiste na principal diferença entre os termos. Logo, pode-se afirmar que nem todos os empreendedores são inovadores, como destacado por alguns conceitos referentes ao empreendedorismo.

Palavras-chave: Empreendedorismo; Inovação; Contexto; Brasil.

\section{Entrepreneurship and innovation: a portrait of the brazilian context of year 20052011}

The themes entrepreneurship and innovation has been much discussed today, both in academic, business and political means. And although they are apparently different concepts, it is suggested that there is a relationship between them. So, in an attempt to verify the existence of this association, this study conducted a qualitative exploratory study, which was used for quantitative data. The survey results show that the terms entrepreneurship and innovation, although some close, express different concepts. Entrepreneurship consists of the process by which individuals turn ideas into opportunities in order to generate wealth. Innovation, in turn, is the process by which new products and services or processes are implemented; Also aims at the continuity of the business and the generation of revenue. Thus the novelty required for innovation is the main difference between terms. Therefore it can be said that not all entrepreneurs are innovators, as highlighted by some concepts related to entrepreneurship.

Keywords: Entrepreneurship; Innovation; Context; Brazil.

\section{Topic: Empreendedorismo}

Reviewed anonymously in the process of blind peer.
Received: 11/10/2017

Approved: 11/12/2017

\section{Suelen Corrêa}

Universidade de Passo Fundo, Brasil

http://lattes.cnpq.br/2699535680870473

sucorrea83@hotmail.com

Neimar Ferreira da Rosa

Faculdade Meridional, Brasil

http://lattes.cnpq.br/8497096409578477

neimarferreira@gmail.com

Eliana Andréa Severo

Universidade Potiguar, Brasil

http://lattes.cnpq.br/0272640518459010

http://orcid.org/0000-0002-5970-4032

elianasevero2@hotmail.com

\section{Hilário Rissardo}

Faculdade Meridional, Brasil

http://lattes.cnpq.br/3636741084875152

hilariorissardo@hotmail.com

Julio Cesar Ferro de Guimarães (iD)

Universidade Federal de Pernambuco, Brasil

http://lattes.cnpq.br/1360508130357781

http://orcid.org/0000-0003-3718-6075

juliocfguimaraes@yahoo.com.br
Referencing this:

CORRÊA, S.; ROSA, N. F.; SEVERO, E. A.; RISSARDO, H.; GUIMARÃES, J. C. F.. Empreendedorismo e inovação: um retrato do contexto brasileiro dos anos de 2005 a 2011. Revista Brasileira de Administração Científica, v.8, n.3, p.99-107, 2017. DOI: http://doi.org/10.6008/SPC2179-684X.2017.003.0008 


\section{INTRODUÇÃO}

Empreendedorismo e inovação são temas em destaque no contexto atual. Relacionados ao crescimento e desenvolvimento econômico, ambos tem estado em evidência nas esferas acadêmica, pública e no mundo dos negócios, em todos os países. Enquanto os acadêmicos têm olhado o empreendedorismo sob o enfoque conceitual, o poder público tem visto no empreendedorismo uma alternativa de renda para os indivíduos, além de reconhecer os negócios como geradores da maior parte dos empregos atuais. Além disso, os pequenos empreendimentos também geram impostos e, assim, contribuem para o giro da economia.

Quanto à inovação, ela tem sido considerada como a grande responsável pelo desenvolvimento econômico, bem como melhoria da performance organizacional, e pode primar para a sustentabilidade ambiental (GUIMARÃES et al., 2016; SEVERO et al.; GUIMARÃES, 2017). E, uma vez que certos tipos de inovação podem se desenvolver em detrimento de investimentos financeiros, consiste em uma grande aliada, inclusive para países pobres e em desenvolvimento.

Mas o que os dois temas, empreendedorismo e inovação, tem em comum? A tentativa de aproximar os dois conceitos, e de identificar relações entre eles, constitui o objetivo deste artigo. A partir de uma leitura das pesquisas que tratam os temas empreendedorismo (GEM, 2005, 2008, 2011) e inovação brasileiros, além da leitura de livros e artigos diversos, foram visitados conceitos que tentam responder a essas questões.

Perante o exposto, no capítulo seguinte é apresentada a introdução deste estudo; o capítulo posterior a esse trata dos conceitos utilizados na pesquisa; por conseguinte, exibe-se o método segundo o qual o estudo foi desenvolvido. O penúltimo capítulo, por sua vez, apresenta a discussão dos assuntos; e, por fim, o último capítulo apresenta as conclusões da pesquisa.

\section{METODOLOGIA}

A pesquisa foi desenvolvida mediante uma abordagem qualitativa e exploratória, e fez uso de dados secundários e quantitativos. A pesquisa qualitativa visa identificar de que maneira e por qual motivo determinados fenômenos acontecem. Caracteriza-se pela subjetividade e interpretação, buscando compreender mais profundamente os eventos (COOPER et al., 2011).

O estudo também pode ser considerado exploratório, método indicado quando o objetivo é obter mais informações e aumentar o entendimento de um determinado assunto, podendo assumir algumas formas, entre elas, a revisão de literatura (HAIR JUNIOR et al., 2005). Para a elaboração desta pesquisa, foram utilizadas fontes de dados secundários, ou seja, os estudos do GEM dos anos de 2005, 2008 e 2011, as PINTECs referentes aos mesmos períodos, além de livros e periódicos diversos.

A análise dos dados foi feita utilizando-se a técnica de análise de conteúdo, cujo objetivo é identificar, organizar, categorizar e, posteriormente, interpretar os dados obtidos através de observações ou texto, evidenciando as principais palavras e temas citados (HAIR JUNIOR et al., 2005). Os dados quantitativos necessários ao estudo foram identificados nas pesquisas GEM e PINTEC, organizados em novas tabelas, calculados seus percentuais e, posteriormente, analisados. 
A pesquisa utilizou, sobretudo, dados quantitativos, uma vez que utiliza números para representar as propriedades de um determinado fato (HAIR JUNIOR et al., 2005), o que possibilita a verificação da sua frequência, além da realização de testes estatísticos (COOPER et al., 2011). Por fim, tentaram-se relacionar os conceitos e informações referentes ao empreendedorismo e à inovação das empresas.

\section{REVISÃO TEÓRICA}

\section{Empreendedorismo}

O empreendedorismo pode ser definido como o envolvimento de pessoas e processos que, em conjunto, levam à transformação de ideias em oportunidades, e a implementação dessas oportunidades resulta na criação dos negócios (DORNELAS, 2014; MUNOZ et al., 2017). O empreendedorismo é entendido como um processo no qual se realiza algo criativo e inovador, objetivando a geração de riqueza e valor para os indivíduos e para a sociedade (SHANE et al., 2000; DORNELAS, 2014).

Nesse contexto, a palavra empreendedor é utilizada para designar principalmente a pessoa que se dedica à geração de riqueza, seja na transformação de conhecimentos em produtos ou serviços, na geração do próprio conhecimento ou na inovação em áreas diversas (DOLABELA, 2008; OMER et al., 2017). Dessa forma, os empreendedores são vislumbrados, geralmente, como atores capazes de produzir e transacionar bens e serviços em um mercado competitivo (SCHUMPETER, 1971).

Quando indivíduos são capazes de reconhecer as oportunidades de negócios no ambiente em que atuam, e perceber que possuem capacidades para explorá-las, toda a sociedade é beneficiada, seja com o aumento da criação de empregos, ou com o aumento da riqueza do país (GEM, 2011; HISRICH et al., 2014). Assim, quando uma sociedade reconhece como um de seus valores a importância das ações referentes à geração de novos negócios, mais indivíduos são sensibilizados pelo empreendedorismo (DOLABELA, 2008).

Para o Global Entrepreneurship Monitor (GEM, 2005), empreendedorismo é o esforço individual ou coletivo na criação de um novo negócio, ou mesmo na expansão de uma empresa já existente. Assim, o empreendedorismo é considerado o propulsor do desenvolvimento das comunidades e da nação.

Com o objetivo de levantar indicadores sobre o empreendedorismo no Brasil, desde o ano 2000 o Brasil passou a fazer parte da pesquisa do GEM, sob a coordenação do Instituto Brasileiro da Qualidade e Produtividade (IBPQ). O GEM tem como principal objetivo o aprimoramento da compreensão sobre a relação do empreendedorismo com o desenvolvimento econômico. A pesquisa classifica as empresas em duas modalidades principais: novos empreendimentos (até 42 meses de existência) e empreendimentos estabelecidos (acima de 42 meses). A pesquisa é realizada anualmente com a população de 18 a 64 anos, composição da força de trabalho (GEM, 2005, 2008, 2011).

O GEM divulga, anualmente, o relatório das pesquisas realizadas em aproximadamente 40 países (35 países em 2005, 43 países em 2008 e 54 países em 2011), cujo objetivo é analisar como a atividade empreendedora se constitui em diferentes partes do mundo. Atualmente, o GEM é administrado pelo Global Entrepreneurship Research (GERA) - organização fundada pela London Business School, no Reino Unido, e pelo Babson College (EUA). 
Em se tratando do Brasil, pode-se observar um crescimento dos índices de empreendedorismo, se considerados os anos de 2005 a 2011 (figura 1), além de um aumento no número de empreendedores iniciais (empreendimentos com até 42 meses de existência), passando de 11,3\% em 2005 para 14,9\% em 2011 (GEM, 2005 , 2011). Além disso, o empreendedorismo por oportunidade no país tem superado o empreendedorismo por necessidade, e se mantido como a principal fonte de criação de novos negócios nos últimos anos (Figura 1). O que sugere que a opção empreendedora tem sido feita mediante um maior preparo e qualificação, em detrimento de se tornar empreendedor por necessidade de uma fonte de renda.

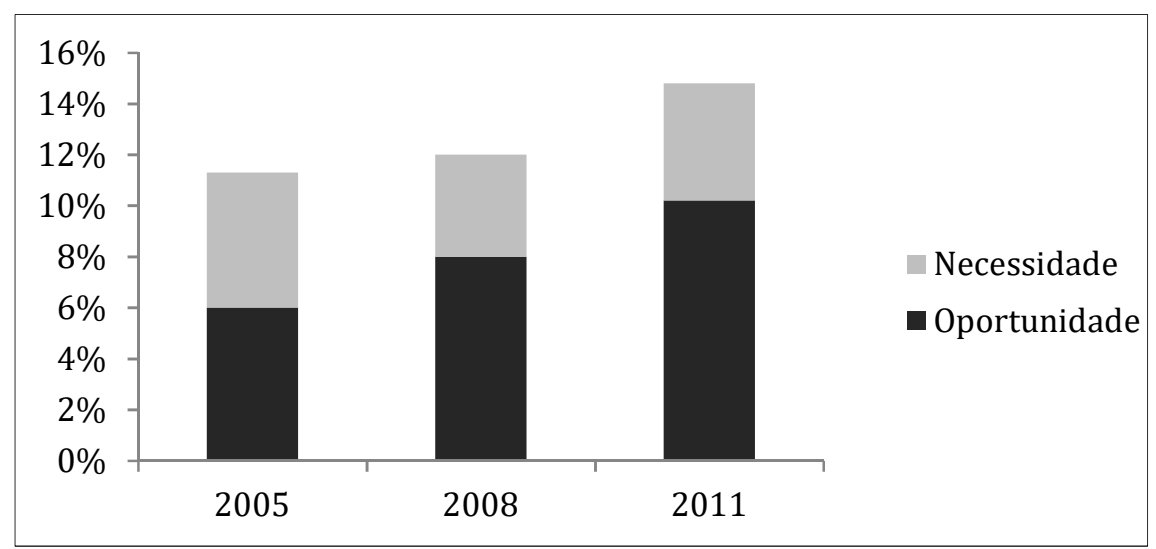

Figura 1: Relação entre empreendedores iniciais, empreendedores por necessidade e oportunidade, de 2005 a 2011. Fonte: GEM $(2005 ; 2008 ; 2011)$.

\section{Inovação}

A inovação consiste numa interessante estratégia a ser utilizada pelas empresas, a fim de se manter e crescer no mercado (GUIMARÃES, et al., 2016; JUNG, 2017). Através das suas várias facetas, concede às organizações a possibilidade de acompanhar as mudanças ocorridas no ambiente, ou, por outro lado, de dirigir e liderar um determinado segmento de mercado (PARK et al., 2017). A inovação não ocorre por sorte ou acaso; pelo contrário, é resultado de um processo deliberado que, a partir dos conhecimentos já adquiridos pela empresa, das informações disponíveis e da criatividade (a novidade), visa obter algo diferente. Deve, além disso, gerar valor social ou riqueza - um produto ou serviço diferenciado que não gera lucro não se caracteriza como uma inovação (TIDD et al., 2008).

Conforme a Organização para Cooperação e Desenvolvimento Econômico (OCDE, 2005), para ser caracterizada como inovação, ela necessita constituir novidade para a empresa, o que permite que ela já seja conhecida pelo mercado. E, além de resultar de um esforço de desenvolvimento interno da empresa, a inovação pode ser obtida de fontes externas, como outras empresas, na forma de aquisição ou licenciamento de tecnologia ou conhecimento, entre outras, o que Chesbrough (2007) chama de inovação aberta.

São quatro os tipos de inovação que uma empresa pode implementar: um novo produto ou serviço, um novo processo, novo método organizacional ou de marketing. A introdução de um novo produto ou serviço no portfólio da empresa, embora já conhecido pelo mercado, constitui uma inovação de produto ou serviço para a organização. Uma inovação de processo consiste na utilização de um novo ou significativamente aperfeiçoado método de produção ou distribuição (OCDE, 2005). Ainda, a mudança na maneira da empresa realizar suas atividades constitui uma inovação organizacional. E, por sua vez, uma 
alteração na embalagem do produto, ou a inserção de um novo canal de distribuição na firma, constitui uma inovação de marketing (OCDE, 2005).

A fim de identificar e mensurar as atividades inovativas das empresas no Brasil, bem como construir indicadores amplos e comparáveis internacionalmente de inovação, desde o ano 2000 o Instituto Brasileiro de Geografia e Estatística (IBGE), com o apoio da Financiadora de Estudos e Projetos (FINEP) e do Ministério da Ciência e Tecnologia (MCT) publica a PINTEC, a Pesquisa de Inovação Brasileira. A pesquisa segue as orientações do Manual de Oslo, referência internacional para pesquisas sobre inovação (OCDE, 2005).

Ao longo das 5 edições, alguns ajustes sobre a PINTEC foram realizados, como a alteração nos setores compreendidos pela pesquisa, bem como seu próprio escopo. Assim, das inovações de produto e processo implementadas unicamente nas indústrias, seu objeto inicial, hoje também fazem parte da pesquisa as inovações de marketing e organizacionais implementadas pelas empresas de comercio e serviços. Para o levantamento dos dados divulgados pela PINTEC, são consultadas empresas com 10 ou mais funcionários, definidas em um processo exaustivo de amostragem.

Entre os anos de 2005 e 2011, o percentual de empresas inovadoras no Brasil apresentou uma variação de 4 pontos percentuais, conforme se visualiza na figura 2. Enquanto no ano de 2005 34,4\% das empresas apresentavam inovações de produto e/ou processo, no ano de 2008 esse índice passou para 38,6\%, passando a cair para $35,7 \%$ no ano de 2011.

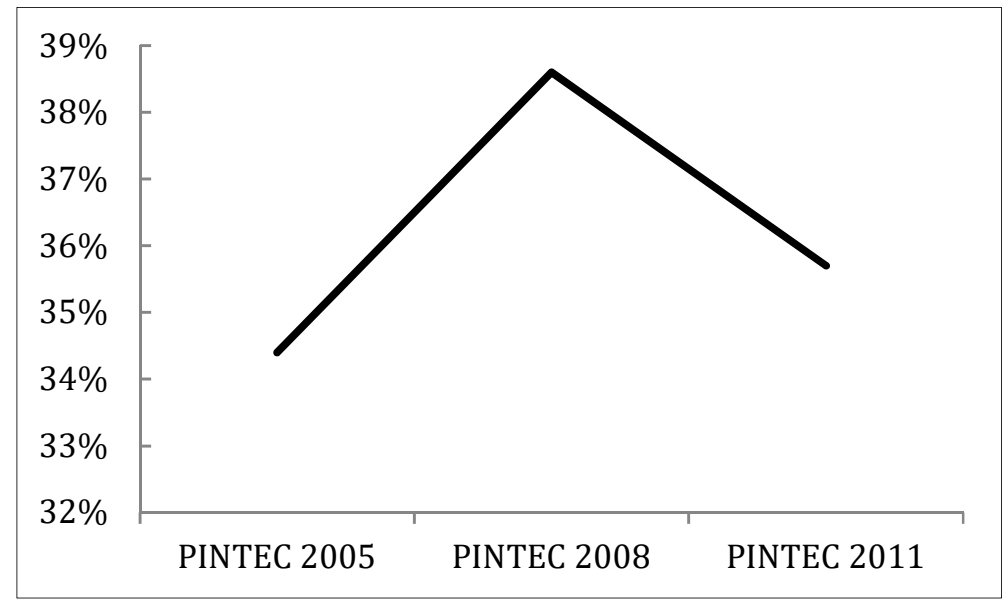

Figura 2: Inovação de produto e/ou processo, de 2005 a 2011, em percentual.

Ainda sobre o mesmo período, os anos de 2005 a 2008, o setor de P\&D foi o que mais implementou inovações em produtos e/ou processos (Tabela 1). Por outro lado, levando-se em conta o setor que mais cresceu na implementação de inovações no período considerado, a Indústria se destaca, passando a representar 38,1\% das empresas que implementaram inovações em 2008, diante de 33,4\% do ano de 2005.

No entanto, no terceiro período apresentado, entre os anos de 2009 e 2011, há uma redução no número de inovações implementadas, tanto no setor da Indústria quanto no de Serviços, embora na Indústria essa redução tenha se mostrado menor, caindo de $38,1 \%$ para $35,6 \%$, enquanto que os Serviços apresentaram uma redução de 9 pontos percentuais.

A tabela 2 demonstra a implementação de inovações de acordo com o tamanho da empresa. Em todo o período considerado, há uma concentração da implementação de inovações nas maiores empresas 
(Tabela 2). Pode-se notar ainda que, de maneira geral, o índice de inovação apresenta um decréscimo significativo em praticamente todos os setores. Do ano de 2005 a 2011, os Serviços Selecionados apresentaram uma redução de 34 pontos percentuais, ao passo que a Indústria apresentou um decréscimo de 23 pontos.

Tabela 1: Empresas, por setor, que implementaram inovações em produto e/ou processo, de 2005 a 2011.

\begin{tabular}{|l|c|c|c|}
\hline & PINTEC 2005 & PINTEC 2008 & PINTEC 2011 \\
\hline Indústria & $33,4 \%$ & $38,10 \%$ & $35,60 \%$ \\
\hline Telecomunicações & $45,9 \%$ & - & - \\
\hline Informática & $57,6 \%$ & - & - \\
\hline Serviços selecionados (1) & - & $46,20 \%$ & $36,80 \%$ \\
\hline P\&D & $97,6 \%$ & $97,50 \%$ & - \\
\hline Eletricidade e gás (2) & - & - & $44,10 \%$ \\
\hline
\end{tabular}

Legenda: (1) Para a PINTEC 2008, os grupos Telecomunicações e Informática foram substituídos pelo grupo de Serviços Selecionados, que compreendem os Serviços de Edição e Gravação de Música, Telecomunicações, Atividades dos Serviços de Tecnologia da Informação, Tratamento de Dados, Hospedagem na Internet e outras atividades relacionadas (2) Para a PINTEC 2011, foi acrescentado o item Eletricidade e Gás.

O único setor que apresentou um incremento na taxa de inovação foi o de $P \& D$, sendo observado nos anos entre 2005 e 2008, já que ele deixou de ser considerado um setor de análise isolado na PINTEC 2011. O setor de Eletricidade e Gás, que passou a integrar a PINTEC em 2011, foi o que apresentou maior taxa de inovação $-82,61 \%$.

Tabela 2: Inovações de produto e/ou processo, por tamanho da empresa, por setor, de 2005 a 2011.

\begin{tabular}{|c|c|c|c|c|c|c|c|c|c|c|c|}
\hline \multirow[b]{2}{*}{ Faixa pessoal ocupado } & \multicolumn{5}{|c|}{2005} & \multicolumn{3}{|c|}{2008} & \multicolumn{3}{|c|}{2011} \\
\hline & Indústria & Telecom. & Informática & P\&D & Serv. Selec. & Indústria & Serv. Selec. & $P \& D$ & Indústria & Serv. Selec. & Eletr. e Gás \\
\hline de 10 a 29 & $28,54 \%$ & - & - & - & $54,66 \%$ & $36,90 \%$ & $45,60 \%$ & $66,70 \%$ & $34,00 \%$ & $32,22 \%$ & $43,91 \%$ \\
\hline de 30 a 49 & $30,80 \%$ & - & - & - & $62,20 \%$ & $35,20 \%$ & $46,50 \%$ & $0,00 \%$ & $33,09 \%$ & $47,42 \%$ & $23,00 \%$ \\
\hline de 10 a 49 (1) & $28,90 \%$ & $36,50 \%$ & $57,30 \%$ & $100,00 \%$ & - & - & - & - & - & - & - \\
\hline de 50 a 99 & $40,60 \%$ & $43,30 \%$ & $53,40 \%$ & $100,00 \%$ & $53,45 \%$ & $40,10 \%$ & $40,80 \%$ & $100,00 \%$ & $38,58 \%$ & $47,82 \%$ & $46,78 \%$ \\
\hline de 100 a 249 & $55,50 \%$ & $83,30 \%$ & $65,30 \%$ & $100,00 \%$ & $70,44 \%$ & $43,00 \%$ & $54,40 \%$ & $100,00 \%$ & $43,43 \%$ & $42,43 \%$ & $52,50 \%$ \\
\hline de 250 a 499 & $65,20 \%$ & $77,80 \%$ & $59,80 \%$ & $100,00 \%$ & $67,54 \%$ & $48,80 \%$ & $56,20 \%$ & $100,00 \%$ & $52,36 \%$ & $40,61 \%$ & $50,00 \%$ \\
\hline com 500 e mais & $79,20 \%$ & $90,90 \%$ & $82,80 \%$ & $91,70 \%$ & $86,14 \%$ & $71,90 \%$ & $67,20 \%$ & $100,00 \%$ & $55,90 \%$ & $52,26 \%$ & $82,61 \%$ \\
\hline Total & $33,40 \%$ & $45,90 \%$ & $57,60 \%$ & $97,60 \%$ & $56,95 \%$ & $38,10 \%$ & $46,20 \%$ & $97,50 \%$ & $35,60 \%$ & $36,80 \%$ & $44,10 \%$ \\
\hline
\end{tabular}

Legenda: A PINTEC 2005 apresenta a taxa de inovação de produto e/ou processo, por setores, somente na faixa das empresas que possuem de 10 a 49 pessoas ocupadas.

Para empresas com 10 a 29 pessoas ocupadas e de 30 a 49, a PINTEC 2005 apresenta o percentual de inovações por serviços selecionados, aglomerando as atividades econômicas.

Parece haver uma aproximação dos temas empreendedorismo e inovação, quando da sua conceituação. Até porque ambos, empreendedorismo e inovação constituem processos que geram riqueza e valor para a sociedade (SHANE et al., 2000; TIDD et al., 2008; DORNELAS, 2014).

Aparentemente, há uma relação direta entre as variações do PIB brasileiro, o número de empreendedores estabelecidos e as inovações de produto e processo nos anos de 2005 a 2011, como pode se notar na figura 3. Logo, aumentos ou reduções no PIB são acompanhados pelos mesmos movimentos nessas variáveis. Quanto ao número de empreendedores iniciais, as variações acompanham os movimentos do PIB nos anos de 2005 a 2008. Contudo, em 2011, há uma relação inversa: enquanto o PIB cai consideravelmente, o número de empreendedores iniciais aumenta, o que pode sugerir que um ambiente econômico menos próspero impele os indivíduos a buscar no empreendedorismo uma oportunidade de renda. 


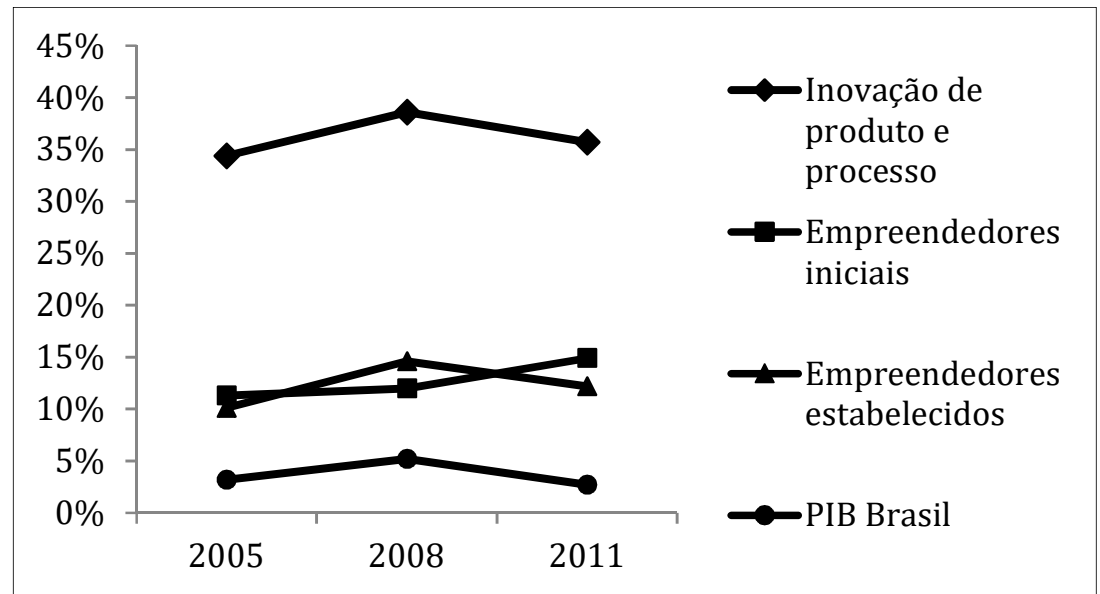

Figura 3: Relação entre PIB, empreendedores iniciais, empreendedores estabelecidos e inovação de produto e processo no Brasil, de 2005 a 2011. Fonte: GEM (2005; 2008; 2011).

Entretanto, apesar de alguns autores usarem o termo inovação já na sua definição de empreendedorismo (VENKATARAMAN, 2000; DORNELAS, 2014), as publicações que tratam de ambos os temas o fazem separadamente (GEM, 2005, 2008, 2011; FIORIN et al., 2010). E a PINTEC, Pesquisa de Inovação Brasileira, nem sequer se refere ao empreendedorismo.

Ademais, as pesquisas do GEM, que utilizam como indicadores de inovação de produto e processo o percentual de empreendedores que lançam produtos desconhecidos pelos consumidores, além de novas tecnologias, tem classificado o Brasil entre os países mais empreendedores; no entanto, estas pesquisas tem demonstrado que o país apresenta baixos índices quando se trata de inovação nos negócios (FIORIN et al., 2010).

Conforme apresentado na pesquisa GEM (2005) para 82,3\% dos empreendedores seus produtos não são considerados novidade pelos clientes; em 2008, este número fica em média 85\%, enquanto que em 2011 esse índice passa a ser de 88,54\%. Esses resultados demonstram que, apesar de haver um amadurecimento do empreendedorismo por oportunidade no Brasil, esses empreendimentos vem se tornando cada vez menos inovadores na percepção dos respondentes da pesquisa.

Contudo, a pesquisa GEM, ao analisar as inovações de produto que constituam novidade para o mercado ou as novas tecnologias envolvidas nos processos, ou seja, as inovações radicais ou incrementais, deixam de considerar a implementação das inovações realmente novas, aquelas que causam descontinuidade de mercado ou de marketing somente para a empresa, e somam aproximadamente 50\% das inovações (GARCIA et al., 2002). Do que se pode depreender que o percentual de inovações implementadas pelos empreendedores é, aproximadamente, o dobro do índice apresentado pela Pesquisa GEM.

\section{CONSIDERAÇÕES FINAIS}

Aproximar os conceitos de empreendedorismo e inovação e verificar a associação entre eles consistiu o objetivo deste estudo. Assim, partindo de uma pesquisa qualitativa, operacionalizada em uma revisão bibliográfica com o uso de dados quantitativos, pretendeu-se analisar os dois conceitos, por meio da obtenção de dados sobre o empreendedorismo e a inovação brasileiros. 
$E$, apesar de alguns autores atrelarem o termo inovação nas suas definições de empreendedorismo (VENKATARAMAN, 2000; DORNELAS, 2014), de acordo com a pesquisa realizada, não se pode afirmar que haja uma relação direta entre eles (GEM, 2005, 2008, 2011; FIORIN et al., 2010). De acordo com os dados obtidos, pode-se afirmar que alguns empreendedores são inovadores, mas não a sua totalidade.

Ademais, o próprio relatório do GEM traz uma pesquisa sobre a inovação dos empreendimentos, o que confirma o argumento de que empreendedorismo e inovação não são sinônimos (GEM, 2005, 2008, 2011). Lembrando, ainda, que a definição de inovação utilizada pelo GEM considera apenas as inovações radicais e as incrementais, descartando $50 \%$ das inovações implementadas pelas empresas (GARCIA et al., 2002).

Quanto à PINTEC, acreditava-se que poderia haver uma relação entre as informações obtidas na pesquisa com os dados sobre empreendedorismo do GEM. Contudo, pelo desenho da amostragem da PINTEC, que entrevista organizações com 10 ou mais funcionários, e que trata a inovação como algo raro, contrário, portanto, às próprias definições do Manual de Oslo (OCDE, 2005), ela deixa de considerar os empreendedores iniciais, que conduzem seus negócios com menos de 10 funcionários.

Deixando de abranger essas empresas, a PINTEC evidencia uma importante lacuna no seu desenho de pesquisa, e impossibilita as aproximações entre os temas empreendedorismo e inovação abordados nas pesquisas GEM e PINTEC. E, haja vista que as micro e pequenas empresas tem uma importância significativa na economia brasileira, uma vez que representam 99\% das empresas registradas, além de gerar 59\% dos empregos formais e cerca de $20 \%$ do PIB em 2013, segundo o SEBRAE, acredita-se que uma importante parcela das empresas tenha sido desconsiderada ao deixar de fazer parte da amostra da PINTEC.

Cabe ressaltar, ainda, que a PINTEC, na sua pesquisa de inovação, interroga empresas de setores bastante específicos, enquanto a GEM faz um levantamento de todo empreendimento de qualquer setor que empregue até 99 funcionários, o que o SEBRAE considera uma pequena empresa. Mais um dado que fortalece $o$ argumento de que as pesquisas não se relacionam.

Assim, a demonstração da diferença dos conceitos de empreendedorismo e inovação, além da lacuna observada na pesquisa PINTEC, que desconsidera os empreendimentos com menos de 10 funcionários, constituem as contribuições do estudo. Entre suas limitações, o fato de ser observado um curto período de tempo, os anos de 2005 a 2011. Nesse contexto, sugerem-se como pesquisas futuras ampliar o período de tempo da análise realizada, bem como a consulta de pesquisas que tratem do Brasil diante das economias emergentes.

\section{REFERÊNCIAS}

CHESBROUGH, H. W.. Why companies should have open business models. MIT Sloan Management Review, v.48, n.2, p.21-28, 2007.

COOPER, D. R.; SCHINDLER, P. S.. Métodos de pesquisa em administração. 10 ed. Porto Alegre: Bookman, 2011.

DOLABELA, F.. Oficina do empreendedor. Rio de Janeiro: Sextante, 2008.
DORNELAS, J.. Empreendedorismo para visionários: desenvolvendo negócios inovadores para um mundo em transformação. Rio de Janeiro: LTC, 2014.

FIORIN, M. M. B.; MELLO, C. M.; MACHADO, H. V.. Empreendedorismo e inovação: análise dos índices de inovação dos empreendimentos brasileiros com base nos 
relatórios do GEM de 2006, 2007 e 2008. Revista de Administração da UFSM, v.3, n.3, p.411-423, 2010.

GARCIA, R.; CALANTONE, R.. A critical look at technological innovation typology and innovativeness terminology: a literature review. The Journal of Product Innovation Management, v.19, n.2, p.110-132, 2002.

GEM. Global Entrepreneurship Monitor. Empreendedorismo no Brasil: 2005. Curitiba: IBQP, 2009.

GEM. Global Entrepreneurship Monitor. Empreendedorismo no Brasil: 2008. Curitiba: IBQP, 2008.

GEM. Global Entrepreneurship Monitor. Empreendedorismo no Brasil: 2011. Curitiba: IBQP, 2011.

GUIMARÃES, J. C. F.; SEVERO, E. A.; DORION, E. C. H.; COALLIER, F.; OLEA, P. M.. The use of organizational resources for product innovation and organizational performance: A survey of the brazilian furniture industry. International Journal of Production Economics, v.180, p.135-147, 2016.

HAIR JUNIOR, J. F.; BABIN, B.; MONEY, A. H.; SAMUEL, P.. Fundamentos de métodos de pesquisa em administração. Porto Alegre: Bookman, 2005.

HISRICH, R. D.; PETERS, M. P.; SHEPHERD, D. A.. Empreendedorismo. 9 ed. Porto Alegre: AMGH, 2014.

JUNG, S. H.. The contingent effect of analyst coverage: how does analyst coverage affect innovation and Tobin's Q?. Asia-Pacific Journal of Accounting \& Economics, v.24, n.1, p.43-67, 2017.
MUNOZ, P.; COHEN, B.. Towards a social-ecological understanding of sustainable venturing. Journal of Business Venturing Insights, v.7, p.1-8, 2017.

OCDE. Organização para a Cooperação e Desenvolvimento Econômico. Manual de Oslo: diretrizes para coleta e interpretação de dados sobre inovação. 3 ed. Paris: OCDE, 2005.

OMER, M. A.; YEMINI, M.. Initiating consensus: stakeholders define entrepreneurship in education. Educational Review, v.69, n.2, p.140-157, 2017.

PARK, K.; KOH, J.. Exploring the relationship between perceived pace of technology change and adoption resistance to convergence products. Computers in Human Behavior, v.69, p.142-150, 2017.

SCHUMPETER, J. A.. Teoria do desenvolvimento econômico: uma investigação sobre lucros, capital, crédito, juro e o ciclo econômico. São Paulo: Abril Cultural, 1971.

SEVERO, E. A.; DORION, E. C. H.; GUIMARAES, J. C. F.. Innovation and environmental sustainability: analysis in Brazilian metal-mechanic industry. International Journal of Innovation and Sustainable Development, v.11, p.230-248, 2017.

SHANE, S.; VENKATARAMAN, S.. The promise of entrepreneurship as a field of research. Academy of Management Review, v.25, n.1, p.217-226, 2000.

TIDD, J.; BESSANT, J.; PAVITT, K.. Gestão da inovação. 3 ed. Porto Alegre: Bookman, 2008. 